El Concepto de Límite Como Una Aproximación Óptima Mediante la Teoría ApOE

\title{
El Concepto de Límite Como Una Aproximación Óptima Mediante la Teoría APOE'
}

\author{
Limit Concept As A Optimal Approximation Theory By APOE
}

Limitar o conceito como uma Teoria da Aproximação Optimal por APOE

Recibido: mayo de 2013

Aprobado: agosto de 2013
Cristian Gonzalo Camacho Ruiz ${ }^{2}$ Jeisson Rodolfo Díaz Martínez ${ }^{3}$

Aymer Alonso Mosquera Herreño ${ }^{4}$ Yina Paola Salamanca Monroy ${ }^{5}$

\section{Resumen}

El presente texto muestra una investigación que trabaja la enseñanza-aprendizaje de aspectos asociados al límite como aproximación optima, desde un análisis teórico (apoyado en APOE) que parte de una descomposición genética del objeto límite y brinda los primeros indicios de las construcciones mentales que poseen los estudiantes, luego se complementa con un parte de diseño e implementación de actividades en el aula con el ciclo de enseñanza ACE. Como la base es una investigación sobre la propia práctica del docente, se trata de un primer avance en este campo, lo que implica un estudio abierto a cualquier persona que requiera ampliarlo y/o complementarlo.

Palabras clave: Aproximación óptima; conocimiento matemático; matemáticas escolares; cálculo; límites; teoría APOE; enseñanza; metodología de enseñanza; metodología de trabajo en el aula.

\begin{abstract}
This paper shows a working research teaching and learning aspects associated to the limit as optimal approach from a theoretical analysis (supported by APOE) that part of a genetic decomposition of the object boundary and provides the first indications of the mental constructs that have students, then complemented by a part of design and implementation of activities in the classroom with the ACE teaching cycle. As the research base is a teacher's practice, it is a first step in this field, which implies an open to any person who requires expansion and / or supplement.
\end{abstract}

Keywords: Best approximation; mathematical knowledge; school math, calculus, limits, APOS theory, teaching, teaching methodology, methodology of work in the classroom.

1 Artículo de Investigación.

2 Universidad Distrital Francisco José de Caldas. Bogotá, Colombia. Contacto: cristiangcamachor@hotmail.com

3 Universidad Distrital Francisco José de Caldas. Bogotá, Colombia. Contacto: jeisson.rdm@gmail.com

4 Universidad Distrital Francisco José de Caldas. Bogotá, Colombia. Contacto: aymermosquera@gmail.com

5 Universidad Distrital Francisco José de Caldas. Bogotá, Colombia. Contacto: paito-48@hotmail.com 


\section{Resumo}

Este trabalho apresenta uma pesquisa de trabalho de ensino e aspectos associados ao limite como melhor abordagem de aprendizagem a partir de uma análise teórica (apoiado por APOE), que parte de uma decomposição genética do limite do objeto e fornece os primeiros indícios das construções mentais que têm estudantes, então, complementada por uma parte do projeto e implementação de atividades em sala de aula com o ciclo de ensino ACE. Como a base de pesquisa é a prática de um professor, é um primeiro passo nesse campo, o que implica um processo aberto a qualquer pessoa que necessite de expansão e / ou suplemento.

Palavras-chave: Melhor aproximação, o conhecimento matemático, matemática do ensino médio, cálculo, limites, teoria APOS, ensino, metodologia de ensino, metodologia de trabalho em sala de aula.

\section{Introducción}

En el proceso de enseñanza de las matemáticas escolares, el Ministerio de Educación Nacional MEN (2006), busca que los estudiantes de educación media desarrollen competencias en relación a la argumentación de procesos de aproximación en rangos de variación y la utilización de técnicas de aproximación en procesos infinitos numéricos. Por ello, en el ciclo de décimo y undécimo grado, se hace necesario vincular objetos matemáticos que trabajen dichos procesos, uno de ellos es el límite, ya que este es un objeto que se relaciona con aproximaciones, convergencia, continuidad, rangos de variación, infinitesimales, entre otros. Pero al abordar la enseñanza y el aprendizaje en la escuela del concepto de límite se presentan algunas dificultades, como:

La primera, como lo menciona Blázquez, Gatica \& Ortega (2008), en la escuela los estudiantes no logran interpretar con facilidad la definición formal de límite y en poco tiempo dicha definición es olvidada; una segunda dificultad está centrada en el proceso de enseñanza que realizan los profesores de matemáticas donde prevalece la ejercitación sin reflexión, por ello, Azcárate, Casadevall, Casellas \& Bosch (1996) afirman que los estudiantes son capaces de realizar ciertos ejercicios sin comprender el significado de límite.

En complemento a lo anterior, Artigue (1998) muestra una tercera dificultad que indica que las concepciones intuitivas de los estudiantes prevalecen aun después de que es presentada la definición formal de límite, por ello, surge la necesidad de buscar formas de relacionar la formalidad del límite con las ideas intuitivas de los estudiantes. De acuerdo a todo lo anterior, se observa una confrontación entre lo que debe ser aprendido-enseñado en la escuela y lo que realmente se da gracias a las dificultades descritas, por ello se hace necesario identificar la comprensión que logran los estudiantes en relación a los procesos de aproximación en rangos de variación, por ello surge la siguiente pregunta de investigación. ¿Cuál es la caracterización del límite como proceso de aproximación optima dentro de las fases de análisis teórico y tratamientos de instrucción de la teoría APOE?

\section{Marco Teorico}

Esta investigación toma como referencia la teoría APOE, por ello para Asiala, Brown, De Vries, Dubinsky, \& Mathews (2004) en la teoría APOE, se indica que:

El conocimiento matemático de un individuo es la tendencia a responder a situaciones problema percibidos por la reflexión sobre los problemas y sus soluciones en un contexto social y por la construcción o reconstrucción de las acciones matemáticas, procesos y objetos y la organización de éstos en esquemas para utilizar en el tratamiento de las situaciones (p.7). 
Además, se indica que comprender en matemáticas, específicamente el concepto de límite como aproximación optima consiste en crear construcciones mentales que actúen sobre situaciones problemas. También, se hace importante (Asiala, Brown, DeVries, Dubinsky, \& Mathews, 2004) para mejorar el conocimiento agregar la reflexión como aquel proceso de tener una atención consciente a las operaciones que se realizan.

En busca de esa reflexión Blázquez, Gatica \& Ortega (2008) brindan una definición que podría ayudar en la comprensión por parte de los estudiantes sobre límite, esta se denomina aproximación óptima y la idea básica de esta definición radica en observar el límite como la mejor aproximación, teniendo en cuenta que dada una aproximación cualquiera (arbitraria) siempre se podrá encontrar una mejor aproximación. De esta manera se tiene que, L es el límite de una sucesión si para cualquier aproximación $\mathrm{K}$ de $\mathrm{L}, \mathrm{K} \neq \mathrm{L}$, existe un término de la sucesión tal que todos los que siguen a este están más próximos a $\mathrm{L}$ que $\mathrm{K}$.

Por otra parte, el análisis teórico que busca como resultado llegar a una descomposición genética de dicho concepto (Asiala, Brown, DeVries, Dubinsky, \& Mathews, 2004). Es decir, la descomposición genética del límite como proceso de aproximación es el conjunto estructurado de construcciones mentales que deben describir cómo se podría desarrollar este concepto en la mente de los estudiantes por ello es importante definir acciones, procesos, objetos y esquemas.

Acciones: de acuerdo con Cottrill, y otros (1996) las acciones son transformaciones físicas o mentales de algún objeto para convertirlo en otro objeto, pero estas transformaciones se presentan como reacción a algo externo, de esta manera puede ser un paso simple para dar una respuesta, un reflejo físico, etc., en general son conocimientos algorítmicos sin reflexión y es un conocimiento muy limitado ya que no muestran un control de lo que se trabaja.

Procesos: de acuerdo con Asiala, Brown, DeVries, Dubinsky, \& Mathews (2004) los procesos suceden cuando se repite una acción y el estudiantes adquiere la capacidad de reflexionar, describir, coordinar con otros procesos o incluso revertir los pasos de la transformación sobre ello y logra tomar el control, sin necesidad de actuar como respuesta a estimulaciones externas.

Objetos: según Alvarenga (2006) los objetos son reconstrucciones de los procesos ya que implican reflexionar sobre las transformaciones (acciones y procesos) como un todo que estructurado conforma objetos cognitivos, se tiene una buena comprensión del objeto matemático y se puede construir, reconstruir, transformar y especificar dicho objeto de manera interna y en algunos casos sin llegar a hacer uso de ejemplos específicos, sino únicamente tratando el concepto en general.

Esquemas: de acuerdo con Asiala, Brown, DeVries, Dubinsky, \& Mathews (2004) un esquema es una colección de procesos y objetos que se organizan de forma estructurada, dichos esquemas pueden ser tratados como objetos y se unen o coordinan con otros esquemas para conformar esquemas de mayor nivel. Para trabajar dichos esquemas se propone seguir lo que indica DeVries (2001, citado en Aldana, 2011) con ello se trabajan 3 niveles, los cuales se denominan intra, inter y trans y lo que diferencia el estar o no en algún nivel no es por el aumento de conocimiento sino por la capacidad de reestructuración y adaptación a un nuevo esquema mental que logra cada individuo al desarrollar cada concepto asociado al límite como aproximación óptima.

\section{Metodología}

La teoría APOE muestra un esquema para el desarrollo de la investigación, se habla de un ciclo con tres fases importantes, 1- el análisis teórico, 2- tratamientos de Instrucción \& 3- evaluación y resultados. El presente artículo trabaja las dos primeras fases con un diseño realizado por estudiantes para profesor, con la intención de aplicarlo en estudiantes de grado 11. Además se tiene en cuenta los aspectos comunes de las investigaciones de tipo cualitativo, entre ellos la recolección de información dentro y fuera de los salones de clase. Por ultimo cabe mencionar que las acciones, procesos, 
objetos y esquemas conforman una espiral que va subiendo de nivel a medida que se llega a construcciones mentales más complejas, cada vez agregando nuevos objetos a esquemas con estructuras cada vez más amplias.

\section{Análisis de Datos}

El análisis teórico. Este análisis se realiza con el objetivo de describir construcciones mentales que deben realizar los estudiantes para desarrollar o entender el concepto de límite como proceso de aproximación. Para empezar las construcciones mentales y la descomposición genética del límite como proceso de aproximación es importante definir acciones, procesos, objetos y esquemas, que se presentan antes de trabajar el concepto en clase, a modo de ejemplo:

Ejemplos de acciones en el límite como proceso de aproximación: - Encontrar un límite reemplazando algún número en alguna fórmula general. Realizar un proceso de aproximación excesivo sin identificar el patrón o regla que permite de manera efectiva (en pocos pasos) encontrar el límite. Ejemplos de procesos en el límite como proceso de aproximación: - Evaluar puntos cercanos para encontrar un patrón o regla para determinar el límite. - Realizar procesos aproximativos que recurran a procesos de generalización para entender el concepto de límite.

Ejemplos de objetos en el límite como proceso de aproximación: - Se logra observar el objeto limite como la mejor aproximación detrás de un proceso de generalización, en el cual se identificó algún tipo de patrón o regla que permitió encontrarlo, además posee un buen significado de la convergencia que lleva consigo la aproximación. Pensar en forma de esquemas sobre los límites como proceso de aproximación implica una comprensión muy cercana del concepto propio de límite y además permite observar este en varias representaciones y sobre todo permite su aplicación en la búsqueda de solucionar situaciones problema que se presenten.
Tratamientos de instrucción. Con el primer boceto del análisis teórico se definen las actividades para llevar al aula y que son planeadas con ACE ciclo de enseñanza (Actividades, discusiones de Clase y Ejercicios), de esta manera, se llevan las actividades al colegio y se genera la reflexión por parte del profesor en busca de mejorar la comprensión del concepto de limite como aproximación optima y los estudiantes de grado 11 generan su propio conocimiento matemático a través de la reflexión sobre la resolución de problemas en un contexto social (empezando por la escuela), tal como lo describe la teoría APOE.

\section{Conclusiones}

Mejorar la comprensión de un objeto matemático es una labor ardua y requiere ser complementada por medio de la práctica misma del profesor de matemáticas, es por ello que se afirma que lo presentado es un trabajo en desarrollo y aún no concluye (se trata de un trabajo hasta el momento teórico y se requiere una parte práctica que aún no se ha realizado), sin embargo deja abierta la posibilidad para aportar en las investigaciones de la teoría APOE, específicamente la enseñanza-aprendizaje del límite. A modo de conclusión parcial, se observa la utilidad de pensar en formas alternas de trabajar los objetos matemáticos dentro del aula, comenzando por la solución de problemas puramente matemáticos hasta una construcción que solo se logra a partir de contextos sociales, por ejemplo las discusiones que se dan en clase al trabajar una hoja de papel que es doblada en mitades de forma recurrente hasta que se determina un límite en ese proceso repetitivo, permitiendo que el objeto matemático límite sea considerado como un objeto propio y no como un algoritmo algebraico sin utilidad.

\section{Referencias}

Alvarenga, K. (2006). Inecuaciones: un análisis de las construcciones mentales de jovenes universitarios. Tesis de Doctorado, Intituto Politécnico Nacional. 
Aldana, E. (2011). Comprensión del concepto de integral definida en el marco de la teoría "APOE". Tesis de Doctorado, Universidad de Salamanca.

Artigue, M. (1998). Enseñanza y aprendizaje del análisis elemental: ¿Qué se puede aprender de las investigaciones didácticas y de los cambios curriculares? Revista latinoamerica de investigación en matemática educativa.

Asiala, M., Brown, A., DeVries, D., Dubinsky, E., \& Mathews, D. (2004). A Framework for Research and Curriculum Development in Undergraduate Mathematics Education. University of Wisconsin-Platteville. Platteville (Wisconsin): RUMEC series.
Blázquez, S., Gatica, S., \& Ortega, T. (2008). Concepto de Limite funcional: Aprendizaje y Memoria. Contextos Educativos, 7-21.

Cottrill, J., Dubinsky, E., Devilyna, N., Schwingendorf, K., Thomas, K., \& Vidakovic, D. (1996). Understanding the Limit Concept: Beginning with a Coordinated Process Schema. Journal of Mathematical Behavior.

MEN. (2006). Estandares básicos de competencias en matemáticas. Bogotá: Magisterio. 